\section{Zehn Jahre Wilsede-Schule für Onkologie und Hämatologie}

\author{
H.J. Illiger
}

10 Jahre Wilsede-Schule geben Anlaß, auf das Erreichte zurückzuschauen und sich auf das $\mathrm{zu}$ besinnen, was deren Gründung stimulierte.

\section{Was will die Wilsede-Schule?}

Die Satzung schreibt schlicht: «Zweck des Vereins ist die Förderung der Wissenschaft und Forschung auf dem Gebiet der Onkologie und Hämatologie». Und weiter: «Der Satzungszweck wird verwirklicht durch Organisation und Durchführung regelmäßiger mehrtägiger Fortbildungsveranstaltungen in Wilsede für junge Wissenschaftler und Mitarbeiter überwiegend onkologisch und hämatologisch tätiger Arbeitsgruppen mit erfahrenen und anerkannten Kennern der klinischen Onkologie und Hämatologie als Dozenten. Dabei soll Gelegenheit geboten werden, wissenschaftlich gesichertes Wissen interdisziplinär aufzuarbeiten und $\mathrm{zu}$ diskutieren sowie neue wissenschaftliche Projekte zu erörtern und $\mathrm{zu}$ realisieren».

\section{Ansprüche von Patienten versus Ansprüche von Disziplinen}

Die Onkologie ist ein interdisziplinäres Querschnittsfach. Die beteiligten Disziplinen (Chirurgische Fächer, Strahlentherapie und medikamentöse Tumortherapie) sind durch Methoden geprägt, nicht durch Organsysteme. Damit steht die Onkologie in gewisser Weise im Widerspruch zu Entwicklungen der Medizin, die die organbezogene Betrachtungsweise von Krankheiten zu stärken suchen.

Onkologie ist eine integrierende ärztliche Disziplin, die Gesundheit und Krankheit als Ganzes erfassen muß und somit einen Arzt verlangt, der Gesundheit und Krankheit auch als Ganzes zu begreifen sucht und der als denkender Arzt am Krankenbett weiß, was er tun und was er lassen muß. Dies aber geht nur auf der Basis einer umfassenden, breiten Weiterbildung und Erfahrung, die ärztliche Qualifikation, Teamfähigkeit und Professionalismus begründen.

Die ärztliche Qualifikation schließt umfassende Kenntnisse der Erkrankungen in ihrer ganzen Komplexität ein, inklusive Differentialdiagnose, Pathophysiologie, Verlauf und Komplikationen, das Beherrschen spezifischer Behandlungsmethoden, deren Nebenwirkungen und Spätfolgen sowie von Begleitsymptomen und Komplikationen. Darüber hinaus sind eingehende Kenntnisse auch von nicht gebiets- und schwerpunktspezifischen Methoden zur Behandlung der Grundkrankheit zu fordern. Sie beinhalten auch die pharmakologischen und nichtpharmakologischen Methoden zur Symptomkontrolle, und Erkennen und Auffangen von psychischem Leid sind gerade in der Onkologie besonders wichtig.

Teamfähigkeit verlangt, daß der den Tumorpatienten betreuende Arzt die Onkologie als multi- und interdisziplinäres Fach versteht und hieraus die Notwendigkeit zur Kooperation ableitet. Dies schließt das Erkennen und Beachten der eigenen Grenzen ebenso ein wie die Achtung und Integration des Könnens von Kollegen anderer Disziplinen. Die aktive Förderung der Kollegialität und die Unterstützung von Teammitgliedern anderer Berufsgruppen sind unabdingbar.

Der Professionalismus bei der Betreuung von Tumorpatienten ist gekennzeichnet durch eine positive Grundeinstellung zur Onkologie, durch Verantwortungsbewußsein für Betroffene sowie Sensitivität für deren Bedürfnisse und Achtung ihrer Anliegen. Die Vertrauenswürdigkeit des Arztes für Patienten und deren Begleiter ist ein besonderes Kriterium für seine Humanität und medizinisch-ethische Grundhaltung. Und «last but not least» sind an der Schwelle zwischen Leben und Tod Wissenschaftlichkeit, Ehrlichkeit und Selbstlosigkeit Kriterien für ethisch sauberen Professionalismus.

Im Rahmen einer kompetenten Betreuung durch jede beteiligte Disziplin erwarten die Patienten Verantwortungsbewußtsein ihnen gegenüber, Sensitivität für ihre Bedürfnisse und Achtung ihrer Anliegen, und sie haben das Recht auf optimale Therapiedurchführung und Schutz vor unbegründeter therapeutischer Aktivität.

Ich hätte mir diese Worte ersparen können, läge in unserem Gesundheitssystem nicht einiges im argen, was es aufzuarbeiten gilt.

Wo liegen die Defizite, die beseitigt werden könnten?

Im Rahmen des Medizinstudiums vermitteln Universitäten im wesentlichen das für den ärztlichen Beruf erforderliche Grundwissen. Bezüglich der Onkologie bestehen aber erhebliche Defizite: Kaum eine Universität bietet eine interdisziplinäre Vorlesung über Onkologie an. Vielmehr wird gerade an medizinischen Fakultäten wie auch an großen Versorgungskrankenhäusern gegenseitige Ausgrenzung verschiedener Disziplinen vorgelebt, und Tugenden, die für die Onkologie essentiell sind und erlernt werden müssen (nämlich die praktische Kooperation), werden kaum vermittelt.

Schon in den 80er Jahren wurde auf einer Jahrestagung der Gesellschaft der Naturforscher und Ärzte prophezeit, daß wahrscheinlich in den letzten 20 Jahren des 20. Jahrhunderts mehr wissenschaftliche Publikationen erscheinen werden als in den 1980 Jahren seit Christi Geburt. Angesichts dieser gigantischen Zunahme des globalen Wissens wächst das individuelle Unwissen. Allein schon aus dieser Tatsache wären Konsequen-

\begin{tabular}{ll}
\hline KARGER & ( 2000 S. Karger GmbH, Freiburg \\
$\begin{array}{l}\text { Fax }+497614520714 \\
\begin{array}{l}\text { E-mail Information@Karger.de } \\
\text { www.karger.com }\end{array}\end{array}$ & $\begin{array}{l}\text { Accessible online at: } \\
\text { www.karger.com/journals/onk }\end{array}$
\end{tabular}

\footnotetext{
Prof. Dr. med. H.J. Illiger

Prof. Dr. med. H.J. Illiger
Städtische Kliniken Oldenburg gGmbH

Klinik für Innere Medizin II - Onkologie/Hämatologie -

Dr.-Eden-Straße 10, D-26133 Oldenburg

Tel. +49 $441403-2611$
} 
zen für eine Änderung der Grundeinstellung zur ärztlichen Tätigkeit abzuleiten, die die Kooperation zum Grundprinzip erheben.

Anfang der 80er Jahre beschäftigte sich der Murrhardter Kreis*, eine von der Robert-Bosch-Stiftung einberufene Arbeitsgruppe von Ordinarien und ärztlichen Standesvertretern, mit einer Reform der ärztlichen Ausbildung, die in ihren Grundsätzen auch auf die ärztliche Weiterbildung übertragen werden muß. Er kritisierte Inhalt und Form der Ausbildung: «Das heutige Medizinstudium vernachlässigt wesentliche Anforderungen an den künftigen Arzt, die heute schon vorauszusehen sind.» Als ein Beispiel werden die alten Menschen mit vielfältigen Krankheiten genannt, die eher eine begleitende als eine kausale Behandlung bräuchten, jedenfalls nicht die Maximaltherapie, die vor allem von jungen Medizinern auf der Basis eines praxisfernen Wissens geprägt wird.

Weiter stellt die Arbeitsgruppe fest, daß «die Fähigkeit zum Erkennen und Lösen komplexer ärztlicher Probleme (neben der Vermittlung isolierten Faktenwissens) schon während des Medizinstudiums gefördert werden muß». Gefordert wird, an die Stelle des statischen, an der Hierarchie der Fächer orientierten medizinischen Lehrgebäudes, das heute die Ärzteausbildung prägt, ein dynamisches, erfahrungsbegründetes Konzept zu setzen, das den Prozeß der Problemlösung und nicht den Wissensbestand in den Mittelpunkt stellt. Die anerkannte Wissenschaftsjournalistin Rosemarie Stein führt in ihrem Kommentar aus: «Dies würde allerdings bedeuten, daß die traditionellen Grenzen zwischen den Fächern und auch zwischen vorklinischer und klinischer Ausbildung überschritten werden müssen. Bereits von Beginn des Studiums an sollten die künftigen Ärzte kritische Selbstbeurteilung, das Erkennen eigener Grenzen und die partnerschaftliche Zusammenarbeit untereinander und mit anderen Berufsgruppen einüben. Statt dessen ist der Medizinstudent unter den heutigen Rahmenbedingungen bestrebt, sich im Alleingang und in Konkurrenz mit anderen möglichst viel Spezialwissen anzueignen. Das fördert Selbstüberschätzung und die Übernahme unbegründeter Entscheidungsautorität, heißt es in den Thesen des Murrhardter Kreises.»

Die Auswirkungen dieser Entwicklung sehen wir heute, und die Onkologie, die des interdisziplinären Dialogs wie kein anderes Krankheitsbild bedarf, leidet hierunter in besonderem Maße.

Wie für Chirurgie und Strahlentherapie gilt aber auch für die medikamentöse Tumortherapie, daß nur eine optimale Primärtherapie die besten Chancen für den Patienten bringen kann. Und eindrückliche Daten von Eurocare über die Heilungschancen von Patienten in unterschiedlichen Ländern zeigen, daß die besten Heilungschancen dort erreicht werden, wo eine Kooperation systematisch aufgebaut und praktiziert wird.

Schon in den 70er Jahren, meinen onkologischen Lehrjahren bei Prof. Dr. G. Martz am Kantonsspital in Zürich, wurde ich mit der Aussage eines Radiotherapeuten konfrontiert, der argwöhnisch den Aufbau einer internistisch onkologischen Abteilung beobachtete und lakonisch (ohne die Probleme des erkrankten Patienten zu beachten) sagte: «Der Krebs gehört mir.» Heute sehen wir ähnliche Anspruchshaltungen der verschiedensten Disziplinen und Schwerpunkte, und die internistische Onkologie ist hier keine Ausnahme.

\section{Die Idee der Wilsede-Schule: Ein bescheidener Ansatz zur Problemlösung}

Mit dem Ziel, diesen negativen Strömungen und Versorgungsproblemen entgegenzuwirken und in der Hoffnung, der Onkologie in Deutschland bei deren Entwicklung zu helfen, gründeten wir die Wilsede-Schule. Sie will jungen Kolleginnen und Kollegen, die in den verschiedenen Disziplinen arbeiten, Wissenschaft unter interdisziplinären Gesichtspunkten vermitteln und in gemütlicher Atmosphäre das gegenseitige Kennenlernen und den gegenseitigen Respekt fördern und eine Basis für Kooperation bei der Patientenversorgung und Forschung schaffen.

In dreitägigen Seminaren werden junge, onkologisch interessierte Ärzte in der Abgeschiedenheit der Lüneburger Heide zusammengeführt, um mit namhaften Dozenten aus dem ganzen Land über die Probleme der Onkologie miteinander zu diskutieren. Dreitägige Seminare mit etwa 40-60 Teilnehmern haben sich nach unseren Erfahrungen als besonders effektiv erwiesen. Die dosierte Steigerung von der Frontvorlesung bis zur aktiven Integration aller Teilnehmer in JournalClubs und Falldiskussionen auf der Basis des vermittelten Wissens fordert jeden Teilnehmer von Beginn an zur wachsamen Teilnahme am Weiterbildungs- bzw. Forbildungsprogramm. Dosierte interaktive Freizeitgestaltung inklusive naturkundlicher Wanderungen sind ein wohlempfundener Stimulus für das positive Erleben des Miteinanders statt einer Aus- und Abgrenzung.

Diese Art der Weiter-/Fortbildung wurde von der WilsedeSchule in den vergangenen 10 Jahren zunächst in ganz kleinen Gruppen im engen Kontakt mit Dozenten aufgebaut und unter Berücksichtigung des Erkenntnisgewinns weiterentwickelt, bis das heutige Gerüst für jede Veranstaltung der Wilsede-Schule zustande kam. Es hat sich bewährt.

\section{Bilanz des Erreichten}

Etwa 1200 Teilnehmer haben die Wilsede-Schule besucht und zirka 190 Referenten haben die Idee der Wilsede-Schule mitgetragen und an der Verwirklichung ihrer Ziele mitgewirkt. Etwa 90 Referenten waren Internisten, zirka 30 waren Radiologen und Radiotherapeuten, jeweils zirka 15 waren Gynäkologen und Medizintheoretiker bzw. Grundlagenforscher, jeweils zirka 8-10 waren Chirurgen, Pathologen und Urologen; Pädiater und Psychologen stellen den fehlenden Rest.

Schon früh übernahmen die Deutsche Krebsgesellschaft und die Deutsche Gesellschaft für Hämatologie und Onkologie die Schirmherrschaft über die Wilsede-Schule. 1993 wurden die Kurse von der European Society for Medical Oncology anerkannt, mit ESMO-MORA-Punkten als zertifizierte Fortbildungen ausgestattet. 1995 wurde die Wilsede-Schule in die European School of Oncology integriert, die europa- und sogar weltweiten Fortbildungsaktivitäten koordiniert und inhaltlich aufeinander abgestimmt. Die Übernahme der Schirmherrschaften und die Integration in die europäischen Aktivitäten empfinde ich als Anerkennung der konstruktiven Zusammenarbeit aller Beteiligten, und hier beziehe ich insbesondere die Seminarleiter ein, die mit hohem Einsatz ihrer 
Arbeitszeit und -kraft die Programme mitgestaltet haben. Diese hohe Anerkennung ehrt ihren Einsatz!

\section{Warum Wilsede?}

Als ich in meiner Hamburger Medizinalassistentenzeit vor über 30 Jahren erstmals in Wilsede war, habe ich diese Landschaft als besonders stimulierend und gleichermaßen erholsam empfunden. Das Eintauchen in eine naturnahe Umgebung, die den Alltag so schnell vergessen läßt, gewährleistet, daß man mit dem Besteigen der Kutschen in Undeloh bereits den Alltag verläßt und empfängnisbereit ist für ein besonderes Erlebnis. Diese Art der Lebensqualität ist das, was wir auch immer für unsere Patienten erreichen möchten. Mehr Lebensqualität bei begrenzter Lebenszeit ist meist unsere Devise für ärztliches Handeln im Interesse unserer Patienten, die nach dem therapeutischen Alltag in der Klinik schnell den Weg in das normale Leben finden sollen. Warum sollen wir nicht auch solche Erlebnisse selbst erfahren, wenn wir im Dienste unserer Patienten unsere Kompetenz entwickeln und pflegen? Entsprechend war die Verknüpfung des Erlebens dieser einmaligen Landschaft nicht nur ein erwünschter Nebeneffekt, den nur dieser Tagungsort mit seiner gewachsenen Tradition vermitteln konnte. Und diesen zu erhalten, gilt es durch aktive
Förderung des Schutzes dieses viel zu kleinen Fleckchens Erde. Mit Unterstützung aller Teilnehmer und Referenten haben wir bei jeder Veranstaltung eine Sammlung zugunsten des Naturschutzes veranstaltet. Auf diese Weise kamen inzwischen mehr als 45000 DM zusammen, die dem Verein Naturschutzpark mit dem Projekt Lüneburger Heide eine wertvolle Unterstützung brachten. Wichtig ist aber auch in Sachen Naturschutz das Miteinander, das wir in Wilsede erlebt haben.

Mein Dank gilt allen Vorsitzenden und Referenten der vergangenen 10 Jahre - nicht nur für ihren unermüdlichen Einsatz vor Ort, sondern auch dafür, daß sie auf jede Honorierung ihrer Arbeit verzichtet und somit eine Basis für ein langfristiges Überleben der Wilsede-Schule gelegt haben. Ihr Einsatz für die Idee der Wilsede-Schule dokumentiert den hohen Idealismus, den sie in ihren Beruf einbringen.

Allerdings wäre der Versuch der Gründung einer deutschsprachigen Onkologieschule ohne finanzielle Unterstützung durch die forschende Industrie sicher gescheitert. Auch ihr also ein besonders herzliches Dankeschön, das ich mit der Hoffnung auf weitere gute Kooperation verknüpfe.

Und ein ganz besonderer Dank gilt meiner langjährigen Mitarbeiterin Frau Schlunk, die die immer wachsende organisatorische Last mit sichtbarer Freude auf sich genommen hat und, obwohl sie inzwischen in den Ruhestand getreten ist, von der Wilsede-Schule nicht ablassen mag. 